\title{
Intuitionistic Anti Fuzzy Vector Spaces with Respect to t-norm and t-conorm
}

\author{
Ongky Denny Wijaya ${ }^{1, *}$ Abdul Rouf Alghofari ${ }^{2}$ Noor Hidayat ${ }^{2}$ \\ ${ }^{1}$ Master's Student of Faculty of Mathematics and Natural Science, Mathematics Department, Brawijaya University, \\ Veteran Street, Malang, East Java, Indonesia \\ ${ }^{2}$ Faculty of Mathematics and Natural Science, Mathematics Department, Brawijaya University, Veteran Street, \\ Malang, East Java, Indonesia \\ *Corresponding author. Email: ongkydenny@gmail.com
}

\begin{abstract}
In this paper, we introduce about intuitionistic anti fuzzy vector spaces with respect to t-norm and t-conorm (IAFVSTC). We discuss some properties of IAFVSTC. We investigate IAFVSTC together with characteristic function intuitionistic fuzzy sets (IFS). We obtain properties of some operation and relation between IAFVSTC, and submodule of $R$-module which the IFS is an IAFVSTC.
\end{abstract}

Keywords: Intuitionistic Fuzzy Sets, t-norm, $t$-conorm, Vector Space.

\section{INTRODUCTION}

The fuzzy sets first time introduced by Zadeh [1] in 1965. Fuzzy sets is generalization of characteristic function, which take the value of 0 or 1 . Fuzzy sets characterized by membership function which associate the elements of non empty sets by the values of $[0,1]$. Fuzzy sets was generalized become intuitionistic fuzzy sets (IFS) by Atanassov [2] in 1981. Intuitionistic fuzzy sets of a non empty sets, characterized by two functions named membership function and non-membership function. Membership function and also nonmembership function take the value of $[0,1]$, and their sum is $[0,1]$. Some operations and relations of IFS was introduced. In 2000, De and Biswas [7] discussed more about some operations and relations of IFS. Schweizer and Sklar [8] have introduced triangular norm and triangular conorm in 1983. In 2012, Sharma [6] researched about intuitionistic anti fuzzy submodules of a module and their properties. In 2013, Rahman and Saikia [4] have introduced about intuitionistic fuzzy submodules with respect to t-norm, and their various properties was investigated. In 2019, Rasuli [5] researching about intuitionistic fuzzy vector space with respect to t-norm and t-conorm, and their properties. In this paper, we introduce about intuitionistic anti fuzzy vector spaces with respect to $\mathrm{t}$-norm and $\mathrm{t}$-conorm
(IAFVSTC). We discuss and investigate some properties of IAFVSTC. We discuss some properties of some operation and relation between IAFVSTC.

\section{PRELIMINARIES}

In this section, we present all basic theory which used in next section.

Definition 2.1. [1] Let $V$ be a vector space over field $F$ and $W$ be a non empty subset of $V$. We shall call $W$ is a subspace of $V$, if the following condition is satisfied:

(1) If $u, v \in V$, then $u+v \in V$.

(2) If $u \in V$ and $a \in F$, then $a u \in V$.

(3) The element 0 of $V$ is also an element of $W$.

Definition 2.2. [1] Let $X$ be non empty set. A fuzzy set $A$ in $X$ is characterized by a membership function $\mu_{A}(x)$ which associates each point in $X$ with a real number in the interval $[0,1]$. In other words, we say the fuzzy sets is a set

$$
A=\left\{\left(x, \mu_{A}(x)\right) \mid x \in X\right\}
$$

which

$$
\mu_{A}: X \rightarrow[0,1]
$$


The value of $\mu_{A}$ at $x$, i.e. $\mu_{A}(x)$ representing the degree of membership of $x$ in $A$.

Definition 2.3. [2] Let $X$ be non empty set. An intuitionistic fuzzy set (IFS) $A$ in $X$ is defined as an object of the following form

$$
A=\left\{\left(x, \mu_{A}(x), v_{A}(x)\right) \mid x \in X\right\}
$$

which $\mu_{A}(x)$ and $v_{A}(x)$ respectively is two functions defined by

$$
\mu_{A}: X \rightarrow[0,1]
$$

and

$$
v_{A}: X \rightarrow[0,1]
$$

which

$$
0 \leq \mu_{A}(x)+v_{A}(x) \leq 1
$$

for every $x \in X$. The functions $\mu_{A}(x)$ and $v_{A}(x)$ define the degree of membership and degree of non-membership respectively, for every $x \in X$.

The operation and relation of IFS is defined as follows.

Definition 2.4. [2, 7] Let $X$ be non empty sets, $A=$ $\left(\mu_{A}, v_{A}\right)$ and $B=\left(\mu_{B}, v_{B}\right)$ are two IFSs on $X$. Relations and operations on IFS defined by

(1) $A \subseteq B$ if and only if $\mu_{A} \leq \mu_{B}$ and $v_{A} \geq v_{B}$.

(2) $A=B$ if and only if $A \subseteq B$ and $B \subseteq A$

(3) $A^{C}=\left\{\left(x, v_{A}(x), \mu_{A}(x)\right) \mid x \in X\right\}$.

(4) $A \cap B=$ $\left\{\left(x, \min \left(\mu_{A}(x), \mu_{B}(x)\right), \max \left(v_{A}(x), v_{B}(x)\right)\right) \mid x \in\right.$ $X\}$.

(5) $A \cup B=$ $\left\{\left(x, \max \left(\mu_{A}(x), \mu_{B}(x)\right), \min \left(v_{A}(x), v_{B}(x)\right)\right) \mid x \in\right.$ $X\}$.

(6) $A+B=\left\{\left(x, \mu_{A}(x)+\mu_{B}(x)-\right.\right.$ $\left.\left.\mu_{A}(x) \mu_{B}(x), v_{A}(x) v_{B}(x)\right) \mid x \in X\right\}$.

(7) $\quad A B=\left\{\left(x, \mu_{A}(x) \mu_{B}(x), v_{A}(x)+v_{B}(x)-\right.\right.$ $\left.\left.v_{A}(x) v_{B}(x)\right) \mid x \in X\right\}$.

(8) $\square A=\left\{\left(x, \mu_{A}(x), 1-\mu_{A}(x)\right) \mid x \in X\right\}$.

(9) $\diamond A=\left\{\left(x, 1-v_{A}(x), v_{A}(x)\right) \mid x \in X\right\}$.

Next, we write about triangular norm and triangular conorm.

Definition 2.5. [4, 5] Let $T$ be a function defined by

$$
T:[0,1] \times[0,1] \rightarrow[0,1] .
$$

$T$ said to be triangular norm (denoted by t-norm) if for all $x, y, z \in[0,1], 4$ axiom below are holds.

(1) Neutral element, i.e. $T(x, 1)=x$.

(2) Monotonicity, i.e. If $y \leq z$ then $T(x, y) \leq T(x, z)$.
(3) Commutativity, i.e. $T(x, y)=T(y, x)$.

(4) Associativity, i.e. $T(x, T(y, z))=T(T(x, y), z)$.

Definition 2.6. [4,5] Let $C$ be a function defined by

$$
C:[0,1] \times[0,1] \rightarrow[0,1] .
$$

$C$ said to be triangular conorm (denoted by t-conorm) if for all $x, y, z \in[0,1], 4$ axiom below are holds.

(1) Neutral element, i.e. $C(x, 0)=x$.

(2) Monotonicity, i.e. If $y \leq z$ then $C(x, y) \leq C(x, z)$.

(3) Commutativity, i.e. $C(x, y)=C(y, x)$.

(4) Associativity, i.e. $C(x, C(y, z))=C(C(x, y), z)$.

Example 2.7. [5] We present the examples of t-norm and t-conorm in Table 1.

Definition 2.8. [4] Let $T$ and $C$ be t-norm and tconorm respectively. We say that $T$ and $C$ are dual if and only if either of

$$
1-T(a, b)=C(1-a, 1-b)
$$

or

$$
1-C(a, b)=T(1-a, 1-b)
$$

are holds, for all $a, b \in[0,1]$.

Example 2.9. [4] This is some examples of t-norm and t-conorm are dual.

(1) Standard intersection t-norm $T_{m}(x, y)=\min (x, y)$ and standard union t-conorm $C_{m}(x, y)=\max (x, y)$ are dual.

(2) Bounded sum t-norm $T_{b}(x, y)=\max (0, x+y-$ 1) and bounded t-conorm $C_{b}(x, y)=\min (1, x+$ y) are dual.

(3) Algebraic product t-norm $T_{p}(x, y)=x y$ and algebraic sum t-conorm $C_{p}(x, y)=x+y-x y$ are dual.

In [5], there are several corollaries about t-norm and t-conorm as follows.

Corollary 2.10. [5] Let $T$ be $t$-norm. Then for all $x \in[0,1]$,

(1) $T(x, 0)=0$.

(2) $T(0,0)=0$

Proof.

(1) Let $x \in(0,1]$. According to Definition 2.5, $T(0,1)=0$. Next we have

$$
0 \leq T(x, 0)=T(0, x) \leq T(0,1)=0 .
$$

(2) Let $x=0$. According to Corollary 2.10 number 1 , we have $T(0,0)=0$. 
Table 1. Example of t-norm and t-conorm.

\begin{tabular}{|c|c|c|}
\hline Name & t-norm & t-conorm \\
\hline $\begin{array}{l}\text { Standard } \\
\text { intersection/ } \\
\text { standard } \\
\text { union }\end{array}$ & $T_{m}(x, y)=\min (x, y)$ & $C_{m}(x, y)=\max (x, y)$ \\
\hline Bounded sum & $T_{b}(x, y)=\max (0, x+y-1)$ & $C_{b}(x, y)=\min (1, x+y)$ \\
\hline $\begin{array}{l}\text { Algebraic product/ } \\
\text { Algebraic sum }\end{array}$ & $T_{p}(x, y)=x y$ & $C_{p}(x, y)=x+y-x y$ \\
\hline Drastic & $T_{D}(x, y)=\left\{\begin{array}{lc}y & \text { if } x=1 \\
x & \text { if } y=1 \\
0 & \text { otherwise }\end{array}\right.$ & $C_{D}(x, y)=\left\{\begin{array}{lc}y & \text { if } x=0 \\
x & \text { if } y=0 \\
1 & \text { otherwise }\end{array}\right.$ \\
\hline $\begin{array}{l}\text { Nilpotent } \\
\text { minimum/ } \\
\text { Nilpotent } \\
\text { maximum }\end{array}$ & $T_{n M}(x, y)=\left\{\begin{array}{cc}\min (x, y) & \text { if } x+y>1 \\
0 & \text { otherwise }\end{array}\right.$ & $C_{n M}(x, y)=\left\{\begin{array}{cc}\max (x, y) & \text { if } x+y<1 \\
1 & \text { otherwise }\end{array}\right.$ \\
\hline $\begin{array}{l}\text { Hamacher } \\
\text { product/ } \\
\text { Einstein sum }\end{array}$ & $T_{H_{0}}(x, y)=\left\{\begin{array}{cc}0 & \text { if } x=y=0 \\
\frac{x y}{x+y-x y} & \text { otherwise }\end{array}\right.$ & $C_{H_{2}}(x, y)=\frac{x+y}{1+x y}$ \\
\hline
\end{tabular}

Corollary 11. [5] Let $C$ be $t$-conorm. Then, for all $x \in[0,1]$,
(1) $\quad C(x, 1)=1$.
(2) $C(0,0)=0$.

Proof.

(1) Let $x \in(0,1]$. According to Definition 2.6, $C(1,0)=1$. Next, we have

$$
1=C(1,0)=C(0,1) \leq C(x, 1) \leq 1 .
$$

Therefore, we can conclude $C(x, 1)=1$.

(2) Obviously from Definition 2.6, we have $C(0,0)=0$.

In [2], the operation intersection and union of two IFSs have been introduced. In [4], they mentioned the operation intersection and union of two IFSs with respect to t-norm and t-conorm as follows.

Definition 2.12. [4] Let $X$ be non empty set. $A=$ $\left(\mu_{A}, v_{A}\right)$ and $B=\left(\mu_{B}, v_{B}\right)$ are two IFSs on $X$. The intersection of $A$ and $B$ with respect to a t-norm and tconorm is defined by

$$
\begin{gathered}
A \cap^{*} B=\left\{\left(x, T\left(\mu_{A}(x), \mu_{B}(x)\right), C\left(v_{A}(x), v_{B}(x)\right)\right) \mid x\right. \\
\in X\} .
\end{gathered}
$$

The union of $A$ and $B$ with respect to a t-norm and tconorm is defined by

$$
\begin{gathered}
A \mathrm{\cup}^{*} B=\left\{\left(x, C\left(\mu_{A}(x), \mu_{B}(x)\right), T\left(v_{A}(x), v_{B}(x)\right)\right) \mid x\right. \\
\in X\} .
\end{gathered}
$$

We denote $\mathrm{O}^{*}$ and $\mathrm{U}^{*}$ for intersection and union of two IFSs with respect to $\mathrm{t}$-norm and $\mathrm{t}$-conorm to distinguish between intersection and union of two IFSs.

Definition 2.13. [5] Let $V$ be vector space over field $F, A=\left(\mu_{A}, v_{A}\right)$ be an intuitionistic fuzzy sets on $V, T$ be t-norm, and $C$ be t-conorm. The IFS $A$ is said to be intuitionistic fuzzy vector spaces with respect to $T$ and $C$ of $V$ (denoted by $A \in \operatorname{IFTC}(V)$ ) if satisfy
(1) $\mu_{A}(x+y) \geq T\left(\mu_{A}(x), \mu_{A}(y)\right)$,
(2) $\mu_{A}(-x) \geq \mu_{A}(x)$,
(3) $\mu_{A}(a x) \geq \mu_{A}(x)$,
(4) $v_{A}(x+y) \leq C\left(v_{A}(x), v_{A}(y)\right)$,
(5) $v_{A}(-x) \leq v_{A}(x)$,
(6) $v_{A}(a x) \leq v_{A}(x)$,

for all $x, y \in V$ and $a \in F$. 


\section{INTUITIONISTIC ANTI FUZZY VECTOR SPACES WITH RESPECT TO T- NORM AND T-CONORM}

In this section, we begin this section with introduce the definition of intuitionistic anti fuzzy vector spaces with respect to t-norm and t-conorm (IAFVSTC). We have several corollaries about IAFVSTC.

Definition 3.1. Let $V$ be vector space over field $F$, $A=\left(\mu_{A}, v_{A}\right)$ be an intuitionistic fuzzy sets on $V, T$ be $\mathrm{t}-$ norm, and $C$ be t-conorm. The IFS $A$ said to be intuitionistic anti fuzzy vector spaces with respect to $T$ and $C$ of $V$ if satisfy

(1) $\mu_{A}(0)=0$

(2) $\mu_{A}(x+y) \leq C\left(\mu_{A}(x), \mu_{A}(y)\right)$,

(3) $\mu_{A}(a x) \leq \mu_{A}(x)$,

(4) $v_{A}(0)=1$,

(5) $v_{A}(x+y) \geq T\left(v_{A}(x), v_{A}(y)\right)$,

(6) $v_{A}(a x) \geq v_{A}(x)$,

for all $x, y \in V$ and $a \in F$. We will denote $A \in$ $\operatorname{IAFVSTC}(V)$ to simplify the intuitionistic anti fuzzy vector space $V$ with respect to $T$ and $C$.

The following is an example of IAFVSTC.

Example 3.2. Given a vector space $\mathbb{R}^{2}$ over field $\mathbb{R}$. Let $A=\left(\mu_{A}, v_{A}\right)$ be an IFS such that

$$
\begin{array}{rll}
\mu_{A}: \mathbb{R}^{2} & \rightarrow \\
\mathbf{x} & \mapsto \quad \mu_{A}(\mathbf{x})= \begin{cases}0 & \mathbf{x}=(0,0) \\
0.25 & \mathbf{x}=(0, b), b \neq 0 \\
0.5 & \mathbf{x}=(a, 0), a \neq 0 \\
0.7 & \text { otherwise }\end{cases}
\end{array}
$$

and

$$
\begin{array}{rll}
v_{A}: \mathbb{R}^{2} & \rightarrow & \\
\mathbf{x} & \mapsto \quad v_{A}(\mathbf{x})= \begin{cases}1 & \mathbf{x}=(0,0) \\
0.6 & \mathbf{x}=(0, b), b \neq 0 \\
0.5 & \mathbf{x}=(a, 0), a \neq 0 \\
0.2 & \text { otherwise }\end{cases}
\end{array}
$$

Given bounded sum t-norm and t-conorm, $T_{b}(x, y)=$ $\max (0, x+y-1)$ and $C_{b}(x, y)=\min (1, x+y)$. Then, $A \in \operatorname{IAFVSTC}\left(\mathbb{R}^{2}\right)$.

Now, we have several corollaries as follows.

Corollary 3.3. If $V$ is vector space over field $F$ and $A=\left(\mu_{A}, v_{A}\right) \in \operatorname{IAFVSTC}(V)$ then for all $x \in V$ satisfies $\mu_{A}(-x) \leq \mu_{A}(x)$ and $v_{A}(-x) \geq v_{A}(x)$.

Proof. Take $x \in V$. Since $V$ is vector space over field $F$, imply $-x \in V$. According to Definition 3.1 we have

$$
\mu_{A}(a(-x))=\mu_{A}((-a) x) \leq \mu_{A}(x) .
$$

Choose $a=1 \in F$ and we obtain

$$
\mu_{A}(-x) \leq \mu_{A}(x) \text {. }
$$

Similarly,

$$
v_{A}(a(-x))=v_{A}((-a) x) \geq v_{A}(x) .
$$

We choose $a=1 \in F$ and now we obtain

$$
v_{A}(-x) \geq v_{A}(x) \text {. }
$$

Corollary 3.4. If $V$ is vector space over field $F$ and $A=\left(\mu_{A}, v_{A}\right) \in \operatorname{IAFVSTC}(V)$ then for all $x \in V$ satisfies $A(-x)=A(x)$.

Proof. We prove using Definition 3.1 as follows.

$$
\begin{gathered}
\mu_{A}(-x) \leq \mu_{A}(x) \quad=\mu_{A}(-(-x)) \leq \mu_{A}(-x), \\
v_{A}(-x) \geq v_{A}(x) \quad=v_{A}(-(-x)) \geq v_{A}(-x) .
\end{gathered}
$$

That explanation show us $\mu_{A}(-x)=\mu_{A}(x)$ and $v_{A}(-x)=v_{A}(x)$. Thus, we have

$A(-x)=\left(\mu_{A}(-x), v_{A}(-x)\right)=\left(\mu_{A}(x), v_{A}(x)\right)=A(x)$.

\section{SOME PROPERTIES OF INTUITIONISTIC ANTI FUZZY VECTOR SPACES WITH RESPECT TO T-NORM AND T-CONORM}

In this section, we discuss and investigate about properties of IAFVSTC.

Theorem 4.1.. Let $V$ be vector space over field $F, W$ be subspace of $V$, and $A=\left(\mu_{A}, v_{A}\right)$ be IFS with $\mu_{A}$ and $v_{A}$ are defined as characteristic function, i.e.

$$
\begin{gathered}
\mu_{A}: V \rightarrow\{0,1\} \\
\mu_{A}(x)= \begin{cases}1 & \text { if } x \in W \\
0 & \text { if } x \notin W\end{cases}
\end{gathered}
$$

and

$$
\begin{gathered}
v_{A}: V \rightarrow\{0,1\} \\
v_{A}(x)= \begin{cases}1 & \text { if } x \in W \\
0 & \text { if } x \notin W\end{cases}
\end{gathered}
$$

If we construct $A_{*}$ as new IFS which defined as $A_{*}=$ $\left(\mu_{A} c, v_{A}\right)$ by

$$
\begin{gathered}
\mu_{A^{C}}: V \rightarrow\{0,1\} \\
\mu_{A^{C}}(x)= \begin{cases}1 & \text { if } x \notin W \\
0 & \text { if } x \in W\end{cases}
\end{gathered}
$$

then $A_{*}=\left(\mu_{A^{C}}, v_{A}\right) \in \operatorname{IAFVSTC}(V)$.

Proof. Take any $x, y \in V$ and $a \in F$. Next, we will divide the proof into three cases as follows.

(1) First case. If $x, y \in W$ then $x+y \in W$ and $a x \in$ $W$. Thus,

(a) $\mu_{A} c(0)=0$.

(b) $\mu_{A}{ }^{c}(x+y)=0 \leq 0=C(0,0)=C\left(\mu_{A} c(x), \mu_{A} c(y)\right)$.

(c) $\mu_{A^{c}}(a x)=0 \leq 0=\mu_{A} c(x)$. 
(d) $v_{A}(0)=1$.

(e) $\quad v_{A}(x+y)=1 \geq 1=T(1,1)=T\left(v_{A}(x), v_{A}(y)\right)$.

(f) $v_{A}(a x)=1 \geq 1=v_{A}(x)$.

(2) Second case. If $x \notin N$ and $y \in N$ then $x+y \notin N$ and $a x \notin N$. Thus,

(a) $\mu_{A} c(0)=0$

(b) $\mu_{A} C(x+y)=1 \leq 1=C(1,0)=C\left(\mu_{A} C(x), \mu_{A} C(y)\right)$

(c) $\mu_{A} c(a x)=1 \leq 1=\mu_{A} c(x)$.

(d) $v_{A}(0)=1$

(e) $v_{A}(x+y)=0 \geq 0=T(0,1)=T\left(v_{A}(x), v_{A}(y)\right)$.

(f) $v_{A}(a x)=0 \geq 0=v_{A}(x)$

(3) Third case. If $x, y \notin N$ then $x+y \notin N$ or $x+y \in$ $N$, and $a x \notin N$. Thus,

(a) $\mu_{A} C(0)=0$

(b) $\mu_{A} C(x+y) \leq 1=C(1,1)=C\left(\mu_{A^{C}}(x), \mu_{A^{C}}(y)\right)$.

(c) $\mu_{A^{c}}(a x)=1 \leq 1=\mu_{A} c(x)$.

(d) $v_{A}(0)=1$.

(e) $\quad v_{A}(x+y) \geq 0=T(0,0)=T\left(v_{A}(x), v_{A}(y)\right)$.

(f) $v_{A}(a x)=0 \geq 0=v_{A}(x)$

Therefore, $A_{*}=\left(\mu_{A} c, v_{A}\right) \in \operatorname{IAFVSTC}(V)$.

Theorem 4.2. If $V$ be a vector space over field $F$ and $A=\left(\mu_{A}, v_{A}\right) \in \operatorname{IAFVSTC}(V)$, then the set

$$
W=\{x \in V \mid A(x)=(0,1)\}
$$

is an subspace of $V$.

Proof. Take $x, y \in W$ and $a \in F$. This gives $\mu_{A}(x)=$ $\mu_{A}(y)=0$ and $v_{A}(x)=v_{A}(y)=1$. Next we show that $W$ is a subspace of $V$ using Definition 2.1. as follows.

Consider that

$$
\begin{aligned}
\mu_{A}(x+y) & \leq C\left(\mu_{A}(x), \mu_{A}(y)\right)=C(0,0)=0 \\
v_{A}(x+y) & \geq T\left(v_{A}(x), v_{A}(y)\right)=T(1,1)=1 .
\end{aligned}
$$

This show us $\mu_{A}(x+y) \leq 0$ and $v_{A}(x+y) \geq 1$. Therefore, $\mu_{A}(x+y)=0$ and $v_{A}(x+y)=1$. Now, we get

$$
A(x+y)=\left(\mu_{A}(x+y), v_{A}(x+y)\right)=(0,1) .
$$

which means that $x+y \in W$.

Consider that

$$
\begin{gathered}
\mu_{A}(a x) \leq \mu_{A}(x)=0 \\
v_{A}(a x) \geq v_{A}(x)=1
\end{gathered}
$$

It means $\mu_{A}(a x)=0$ and $v_{A}(a x)=1$. Next, we have

$$
A(a x)=\left(\mu_{A}(a x), v_{A}(a x)\right)=(0,1),
$$

which means that $a x \in W$.

Take $0 \in V$. We can see that

$$
A(0)=\left(\mu_{A}(0), v_{A}(0)\right)=(0,1) .
$$

It means $0 \in W$.

Therefore, $W$ is a subspace of $V$.

Theorem 4.3. Let $V$ be vector space over field $F$ and $A=\left(\mu_{A}, v_{A}\right) \in \operatorname{IAFVSTC}(V)$. If $A(x-y)=A(0)$ then $A(x)=A(y)$, for all $x, y \in V$.

Proof. Take $x, y \in V$. Given that $\mu_{A}(x-y)=\mu_{A}(0)$ and $v_{A}(x-y)=v_{A}(0)$. We will show $\mu_{A}(x)=\mu_{A}(y)$ and $v_{A}(x)=v_{A}(y)$. Consider that

$$
\begin{aligned}
\mu_{A}(x) & =\mu_{A}(x-y+y) \\
& \leq C\left(\mu_{A}(x-y), \mu_{A}(y)\right) \\
& =C\left(\mu_{A}(0), \mu_{A}(y)\right) \\
& =C\left(0, \mu_{A}(y)\right) \\
& =\mu_{A}(y)
\end{aligned}
$$

and

$$
\begin{aligned}
\mu_{A}(y) & =\mu_{A}(x-x+y) \\
& =\mu_{A}(x-(x-y)) \\
& \leq C\left(\mu_{A}(x), \mu_{A}(-(x-y))\right) \\
& =C\left(\mu_{A}(x), \mu_{A}(x-y)\right) \\
& =C\left(\mu_{A}(x), \mu_{A}(0)\right) \\
& =C\left(\mu_{A}(x), 0\right) \\
& =\mu_{A}(x) .
\end{aligned}
$$

Thus, we have $\mu_{A}(x)=\mu_{A}(y)$. Similarly,

$$
\begin{aligned}
v_{A}(x) & =v_{A}(x-y+y) \\
& \geq T\left(v_{A}(x-y), v_{A}(y)\right) \\
& =T\left(v_{A}(0), v_{A}(y)\right) \\
& =T\left(1, v_{A}(y)\right) \\
& =v_{A}(y)
\end{aligned}
$$

and

$$
\begin{aligned}
v_{A}(y) & =v_{A}(x-x+y) \\
& =v_{A}(x-(x-y)) \\
& \leq T\left(v_{A}(x), v_{A}(-(x-y))\right) \\
& =T\left(v_{A}(x), v_{A}(x-y)\right) \\
& =T\left(v_{A}(x), v_{A}(0)\right) \\
& =T\left(v_{A}(x), 1\right) \\
& =v_{A}(x) .
\end{aligned}
$$

We have $v_{A}(x)=v_{A}(y)$. Thus,

$$
A(x)=\left(\mu_{A}(x), v_{A}(x)\right)=\left(\mu_{A}(y), v_{A}(y)\right)=A(y) .
$$

Theorem 4.4. Let $V$ be vector space over field $F$ and $A=\left(\mu_{A}, v_{A}\right) \in \operatorname{IAFVSTC}(V)$. If $A(x-y)=(0,1)$ then $A(x)=A(y)$, for all $x, y \in V$.

Proof. Given that $\mu_{A}(x-y)=0$ and $v_{A}(x-y)=$ 1. Consider that

$$
\begin{aligned}
\mu_{A}(x) & =\mu_{A}(x-y+y) \\
& \geq C\left(\mu_{A}(x-y), \mu_{A}(y)\right)
\end{aligned}
$$




$$
\begin{aligned}
& =C\left(0, \mu_{A}(y)\right) \\
& =\mu_{A}(y)
\end{aligned}
$$

and

$$
\begin{aligned}
\mu_{A}(y) & =\mu_{A}(x-x+y) \\
& =\mu_{A}(x-(x-y)) \\
& \geq C\left(\mu_{A}(x), \mu_{A}(-(x-y))\right) \\
& =C\left(\mu_{A}(x), \mu_{A}(x-y)\right) \\
& =C\left(\mu_{A}(x), 0\right) \\
& =\mu_{A}(x) .
\end{aligned}
$$

Now, we have $\mu_{A}(x)=\mu_{A}(y)$. Similarly,

$$
\begin{aligned}
v_{A}(x) & =v_{A}(x-y+y) \\
& \leq T\left(v_{A}(x-y), v_{A}(y)\right) \\
& =T\left(1, v_{A}(y)\right) \\
& =v_{A}(y)
\end{aligned}
$$

and

$$
\begin{aligned}
v_{A}(y) & =v_{A}(x-x+y) \\
& =v_{A}(x-(x-y)) \\
& \geq T\left(v_{A}(x), v_{A}(-(x-y))\right) \\
& =T\left(v_{A}(x), v_{A}(x-y)\right) \\
& =T\left(v_{A}(x), 1\right) \\
& =v_{A}(x) .
\end{aligned}
$$

Thus, we have $v_{A}(x)=v_{A}(y)$. Now, it is leads to

$$
A(x)=\left(\mu_{A}(x), v_{A}(x)\right)=\left(\mu_{A}(y), v_{A}(y)\right)=A(y) .
$$

\section{SOME PROPERTIES OF OPERATION AND RELATION BETWEEN INTUITIONISTIC ANTI FUZZY VECTOR SPACES WITH RESPECT TO T-NORM AND T-CONORM}

In this section, we investigate and prove some properties of operation and relation about IAFVSTC.

Theorem 5.1. If $V$ be vector space over field $F$ and $A=\left(\mu_{A}, v_{A}\right), B=\left(\mu_{B}, v_{B}\right) \in \operatorname{IAFVSTC}(V)$, then $A \cup^{*} B \in \operatorname{IAFVSTC}(V)$.

Proof. We prove this theorem according to Definition 2.12 as follows.

(1) $\mu_{A \cup^{*} B}(0)=C\left(\mu_{A}(0), \mu_{B}(0)\right)=C(0,0)=0$.

(2) $\mu_{A U^{*} B}(x+y)=C\left(\mu_{A}(x+y), \mu_{B}(x+y)\right)$

$$
\begin{aligned}
& \leq C\left(C\left(\mu_{A}(x), \mu_{A}(y)\right), C\left(\mu_{B}(x), \mu_{B}(y)\right)\right) \\
& =C\left(C\left(\mu_{A}(x), \mu_{B}(x)\right), C\left(\mu_{A}(y), \mu_{B}(y)\right)\right) \\
& =C\left(\mu_{A \cup^{*} B}(x), \mu_{A \cup^{*} B}(y)\right)
\end{aligned}
$$

(3) $\mu_{A U^{*} B}(a x)=C\left(\mu_{A}(a x), \mu_{B}(a x)\right)$

$$
\begin{aligned}
& \leq C\left(\mu_{A}(x), \mu_{B}(x)\right) \\
& =\mu_{A \cup^{*} B}(x)
\end{aligned}
$$

(4) $v_{A \cup^{*} B}(0)=T\left(v_{A}(0), v_{B}(0)\right)=T(1,1)=1$.

(5) $v_{A \cup^{*} B}(x+y)=T\left(v_{A}(x+y), v_{B}(x+y)\right)$

$$
\begin{aligned}
& \geq T\left(T\left(v_{A}(x), v_{A}(y)\right), T\left(v_{B}(x), v_{B}(y)\right)\right) \\
& =T\left(T\left(v_{A}(x), v_{B}(x)\right), T\left(v_{A}(y), v_{B}(y)\right)\right) \\
& =T\left(v_{A \cup^{*} B}(x), v_{A \cup^{*} B}(y)\right)
\end{aligned}
$$

(6) $v_{A \cup^{*} B}(a x)=T\left(v_{A}(a x), v_{B}(a x)\right)$

$$
\begin{aligned}
& \geq T\left(v_{A}(x), v_{B}(x)\right) \\
& =v_{A \cup^{*} B}(x)
\end{aligned}
$$

Therefore $A \cup^{*} B \in \operatorname{IAFVSTC}(V)$.

The next theorem is generalization of Theorem 5.1.

Theorem 5.2. If $V$ be vector space over field $F$ and $A_{i}=\left(\mu_{A_{i}}, v_{A_{i}}\right) \in \operatorname{IAFVSTC}(V)$, for $i=1,2, \ldots, n$ and $n \in \mathbb{N}$, then

$$
\bigcup_{i=1}^{n} * A_{i} \in \operatorname{IAFVSTC}(V) .
$$

Proof. The proof using mathematical induction.

Theorem 5.3. Let $V$ be vector space over field $F$, $A=\left(\mu_{A}, v_{A}\right), B=\left(\mu_{B}, v_{B}\right) \in \operatorname{IAFVSTC}(V)$, and $T$ and $C$ be t-norm and t-conorm respectively. If $T$ and $C$ are duals, then $\square A \in I A F V S T C(V)$.

Proof. Take $x, y \in V$ and $a \in F$.

$$
\begin{aligned}
& \mu_{\square A}(0)=\mu_{A}(0)=0 \\
& \mu_{\square A}(x+y)=\mu_{A}(x+y) \\
& \leq C\left(\mu_{A}(x), \mu_{A}(y)\right) \\
& =C\left(\mu_{\square A}(x), \mu_{\square A}(y)\right) \\
& \geq 1-C\left(\mu_{A}(x), \mu_{A}(y)\right) \\
& =T\left(1-\mu_{A}(x), 1-\mu_{A}(y)\right) \\
& =T\left(v_{\square A}(x), v_{\square A}(y)\right)
\end{aligned}
$$

(6) $\quad v_{\square A}(a x)=1-\mu_{A}(a x) \geq 1-\mu_{A}(x)=v_{\square A}(x)$

Thus, $\square A \in \operatorname{IAFVSTC}(V)$.

Theorem 5.4. Let $V$ be vector space over field $F$, $A=\left(\mu_{A}, v_{A}\right), B=\left(\mu_{B}, v_{B}\right) \in \operatorname{IAFVSTC}(V)$, and $T$ and $C$ be t-norm and t-conorm respectively. If $T$ and $C$ are duals, then $\diamond A \in \operatorname{IAFVSTC}(V)$.

Proof. Take $x, y \in V$ and $a \in F$.

(1) $\mu_{\diamond A}(0)=1-v_{A}(0)=1-1=0$.

(2) $\mu_{\diamond A}(x+y)=1-v_{A}(x+y)$

$$
\begin{aligned}
& \leq 1-T\left(v_{A}(x), v_{A}(y)\right) \\
& =C\left(1-v_{A}(x), 1-v_{A}(y)\right) \\
& =C\left(\mu_{\diamond A}(x), \mu_{\diamond A}(y)\right)
\end{aligned}
$$


(3) $\mu_{\diamond A}(a x)=1-v_{A}(a x) \leq 1-v_{A}(x)=\mu_{\diamond A}(x)$

(4) $v_{\diamond A}(0)=v_{A}(0)=1$

(5) $v_{\diamond A}(x+y)=v_{A}(x+y)$

$$
\begin{aligned}
& \geq T\left(v_{A}(x), v_{A}(y)\right) \\
& =T\left(v_{\diamond A}(x), v_{\diamond A}(y)\right)
\end{aligned}
$$

(6) $v_{\diamond A}(a x)=v_{A}(a x) \geq v_{A}(x)=v_{\diamond A}(x)$.

Thus, $\diamond A \in \operatorname{IAFVSTC}(V)$.

Theorem 5.5. Let $V$ be vector space over field $F$ and $A=\left(\mu_{A}, v_{A}\right), B=\left(\mu_{B}, v_{B}\right) \in \operatorname{IAFVSTC}(V)$. If $\operatorname{IAFVSTC}(V)$ have t-norm $T$ which satisfied the condition

$$
\begin{gathered}
T\left(v_{A}(x), v_{A}(y)\right)+T\left(v_{B}(x), v_{B}(y)\right) \\
-T\left(v_{A}(x), v_{A}(y)\right) T\left(v_{B}(x), v_{B}(y)\right) \\
\geq T\left(v_{A}(x)+v_{B}(x)-v_{A}(x) v_{B}(x), v_{A}(y)+v_{B}(y)\right. \\
\left.-v_{A}(y) v_{B}(y)\right)
\end{gathered}
$$

and t-conorm $C$ which satisfied the condition

$$
\begin{aligned}
& C\left(\mu_{A}(x), \mu_{A}(y)\right) C\left(\mu_{B}(x), \mu_{B}(y)\right) \\
& \leq C\left(\mu_{A}(x) \mu_{B}(x), \mu_{A}(y) \mu_{B}(y)\right)
\end{aligned}
$$

for all $x, y \in V$ then $A B \in \operatorname{IAFVSTC}(V)$.

Proof. Take any $x, y \in V$ and $a \in F$.

(1) $\mu_{A B}(0)=\mu_{A}(0) \mu_{B}(0)=0 \cdot 0=0$.

(2) $\mu_{A B}(x+y)=\mu_{A}(x+y) \mu_{B}(x+y)$

$$
\begin{aligned}
& \leq C\left(\mu_{A}(x), \mu_{A}(y)\right) C\left(\mu_{B}(x), \mu_{B}(y)\right) \\
& \leq C\left(\mu_{A}(x) \mu_{B}(x), \mu_{A}(y) \mu_{B}(y)\right) \\
& =C\left(\mu_{A B}(x), \mu_{A B}(y)\right)
\end{aligned}
$$

(3) $\mu_{A B}(a x)=\mu_{A}(a x) \mu_{B}(a x)$

$$
\leq \mu_{A}(x) \mu_{B}(x)=\mu_{A B}(x) \text {. }
$$

(4) $v_{A B}(0)=v_{A}(0)+v_{B}(0)-v_{A}(0) v_{B}(0)$

$$
=1+1-1 \cdot 1=1 \text {. }
$$

(5) $v_{A B}(x+y)=v_{A}(x+y)+v_{B}(x+y)-v_{A}(x+$ y) $v_{B}(x+y)$

$\geq T\left(v_{A}(x), v_{A}(y)\right)+T\left(v_{B}(x), v_{B}(y)\right)$

$-T\left(v_{A}(x), v_{A}(y)\right) T\left(v_{B}(x), v_{B}(y)\right)$

$\geq T\left(v_{A}(x)+v_{B}(x)-v_{A}(x) v_{B}(x), v_{A}(y)+v_{B}(y)\right.$

$$
=T\left(v_{A B}(x), v_{A B}(y)\right)
$$

$$
\left.-v_{A}(y) v_{B}(y)\right)
$$

(6) $v_{A B}(a x)=v_{A}(a x)+v_{B}(a x)-v_{A}(a x) v_{B}(a x)$

$$
\begin{aligned}
& \geq v_{A}(x)+v_{B}(x)-v_{A}(x) v_{B}(x) \\
& =v_{A B}(x)
\end{aligned}
$$

So, $A B \in \operatorname{IAFVSTC}(V)$.

Theorem 5.6. Let $V$ be vector space over field $F$ and $A=\left(\mu_{A}, v_{A}\right), B=\left(\mu_{B}, v_{B}\right) \in \operatorname{IAFVSTC}(V)$. If $\operatorname{IAFVSTC}(V)$ have t-norm $T$ which satisfied the condition

$$
\begin{aligned}
& T\left(v_{A}(x), v_{A}(y)\right) T\left(v_{B}(x), v_{B}(y)\right) \\
& \geq T\left(v_{A}(x) v_{B}(x), v_{A}(y) v_{B}(y)\right)
\end{aligned}
$$

and t-conorm $C$ which satisfied the condition

$$
\begin{gathered}
C\left(\mu_{A}(x), \mu_{A}(y)\right)+C\left(\mu_{B}(x), \mu_{B}(y)\right) \\
-C\left(\mu_{A}(x), \mu_{A}(y)\right) C\left(\mu_{B}(x), \mu_{B}(y)\right) \\
\leq C\left(\mu_{A}(x)+\mu_{B}(x)-\mu_{A}(x) \mu_{B}(x), \mu_{A}(y)+\mu_{B}(y)\right. \\
\left.-\mu_{A}(y) \mu_{B}(y)\right)
\end{gathered}
$$

for all $x, y \in V$ then $A+B \in \operatorname{IAFVSTC}(V)$.

Proof. Let $x, y \in V$ and $a \in F$.

(1) $\mu_{A+B}(0)=\mu_{A}(0)+\mu_{B}(0)-\mu_{A}(0) \mu_{B}(0)=0+$ $0-0 \cdot 0=0$.

(2) $\mu_{A+B}(x+y)=\mu_{A}(x+y)+\mu_{B}(x+y)-\mu_{A}(x+$ y) $\mu_{B}(x+y)$

$$
\begin{aligned}
& \leq C\left(\mu_{A}(x), \mu_{A}(y)\right)+C\left(\mu_{B}(x), \mu_{B}(y)\right) \\
& -C\left(\mu_{A}(x), \mu_{A}(y)\right) C\left(\mu_{B}(x), \mu_{B}(y)\right) \\
& \leq C\left(\mu_{A}(x)+\mu_{B}(x)-\mu_{A}(x) \mu_{B}(x), \mu_{A}(y)\right. \\
& \left.\quad+\mu_{B}(y)-\mu_{A}(y) \mu_{B}(y)\right) \\
& =C\left(\mu_{A+B}(x), \mu_{A+B}(y)\right) .
\end{aligned}
$$

(3) $\mu_{A+B}(a x)=\mu_{A}(a x)+\mu_{B}(a x)-\mu_{A}(a x) \mu_{B}(a x)$

$$
\begin{aligned}
& \leq \mu_{A}(x)+\mu_{B}(x)-\mu_{A}(x) \mu_{B}(x) \\
& =\mu_{A+B}(x) .
\end{aligned}
$$

(4) $v_{A+B}(0)=v_{A}(0) v_{B}(0)=1 \cdot 1=1$.

(5) $v_{A+B}(x+y)=v_{A}(x+y) v_{B}(x+y)$

$$
\begin{aligned}
& \geq T\left(v_{A}(x), v_{A}(y)\right) T\left(v_{B}(x), v_{B}(y)\right) \\
& \geq T\left(v_{A}(x) v_{B}(x), v_{A}(y) v_{B}(y)\right) \\
& =T\left(v_{A+B}(x), v_{A+B}(y)\right) .
\end{aligned}
$$

(6) $v_{A+B}(a x)=v_{A}(a x) v_{B}(a x)$

$$
\begin{aligned}
& \geq v_{A}(x) v_{B}(x) \\
& =v_{A+B}(x) .
\end{aligned}
$$

So, $A+B \in \operatorname{IAFVSTC}(V)$.

Corollary 5.7. Let $V$ be vector space over $F$ and $A=$ $\left(\mu_{A}, v_{A}\right), B=\left(\mu_{B}, v_{B}\right) \in \operatorname{IAFVSTC}(V)$. If $T$ is algebraic product t-norm, i.e. $T_{p}(a, b)=a b$ and $C$ is algebraic sum t-conorm, i.e. $C_{p}(a, b)=a+b-a b$, for all $a, b \in$ $[0,1]$ then $A+B \in \operatorname{IAFVSTC}(V)$.

Proof. Consider that

$$
T_{p}(a, b) T_{p}(c, d)=T_{p}(a c, b d)
$$

and

$$
\begin{aligned}
C_{p}(a, b)+C_{p}(c, d)-C_{p}(a, b) C_{p}(c, d) & \\
& =C_{p}(a+c-a c, b+d-b d),
\end{aligned}
$$

for any $a, b, c, d \in[0,1]$. This implies

$$
\begin{aligned}
T\left(v_{A}(x), v_{A}(y)\right) T & \left(v_{B}(x), v_{B}(y)\right) \\
= & T\left(v_{A}(x) v_{B}(x), v_{A}(y) v_{B}(y)\right)
\end{aligned}
$$

and

$$
\begin{gathered}
C\left(\mu_{A}(x), \mu_{A}(y)\right)+C\left(\mu_{B}(x), \mu_{B}(y)\right) \\
-C\left(\mu_{A}(x), \mu_{A}(y)\right) C\left(\mu_{B}(x), \mu_{B}(y)\right) \\
=C\left(\mu_{A}(x)+\mu_{B}(x)-\mu_{A}(x) \mu_{B}(x), \mu_{A}(y)+\mu_{B}(y)\right. \\
\left.-\mu_{A}(y) \mu_{B}(y)\right) .
\end{gathered}
$$


for any $x, y \in V$. According to Theorem 5.6, $A+B \in$ $\operatorname{IAFVSTC}(V)$.

Theorem 5.8. Let $V$ be vector space over field $F$. If $A=\left(\mu_{A}, v_{A}\right) \in \operatorname{IAFVSTC}(V)$ then $A^{C}=\left(\mu_{A} C, v_{A} C\right)=$ $\left(v_{A}, \mu_{A}\right) \in \operatorname{IFTC}(V)$.

Proof. Let $A=\left(\mu_{A}, v_{A}\right) \in \operatorname{IAFVSTC}(M)$. Take $x, y \in V$ and $a \in F$. Consider that

(1) $\mu_{A} c(x+y)=v_{A}(x+y)$

$$
\begin{aligned}
& \geq T\left(v_{A}(x), v_{A}(y)\right) \\
& =T\left(\mu_{A} c(x), \mu_{A^{C}}(y)\right) .
\end{aligned}
$$

(2) $\mu_{A}{ }^{C}(-x)=v_{A}(-x) \geq v_{A}(x)=\mu_{A} c(x)$.

(3) $\mu_{A} c(a x)=v_{A}(a x) \geq v_{A}(x)=\mu_{A} c(x)$.

(4) $v_{A} C(x+y)=\mu_{A}(x+y)$

$$
\leq C\left(\mu_{A}(x), \mu_{A}(y)\right)=C\left(v_{A} C(x), v_{A^{C}}(y)\right) .
$$

(5) $v_{A} C(-x)=\mu_{A}(-x) \leq \mu_{A}(x)=v_{A} c(x)$.

(6) $v_{A} c(a x)=\mu_{A}(a x) \leq \mu_{A}(x)=v_{A} c(x)$.

Thus, $A^{C}=\left(\mu_{A} C, v_{A^{C}}\right)=\left(v_{A}, \mu_{A}\right) \in \operatorname{IFTC}(V)$.

\section{CONCLUSION}

We have proved several properties related with intuitionistic anti fuzzy vector space with respect to tnorm and t-conorm (IAFVSTC) and some operation and relation between IAFVSTC.

\section{ACKNOWLEDGMENTS}

We give special thanks to the reviewer for reviewing this article and giving suggestions and comments.

\section{REFERENCES}

[1] L.A. Zadeh, Fuzzy Sets, Journal of Information and Control, 1965 , vol. 8 pp. 338-353

[2] K.T. Atanassov, Intuitionistic Fuzzy Sets Bulgaria: Springer-Verlag, 1999

[3] S. Lang, Linear Algebra Second Edition New York: Addison-Wesley, 1972

[4] S. Rahman and H.K. Saikia, Atanassov's Intuitionistic Fuzzy Submodules with respect to a tnorm. Soft Computing, 2013, vol. 17 pp. 1253-1262

[5] R. Rasuli Norms Over Intuitionistic Fuzzy Vector Space Algebra Letters, 2019, pp. 1

[6] P.K. Sharma, On Intuitionistic Anti-Fuzzy Submodule of a Module Int. J. of Fuzzy Mathematics and Systems, 2012, vol. 2 pp. 127-132

[7] S.K. De and R. Biswas, Some Operations on Intuitionistic Fuzzy Sets J. Fuzzy Sets and Systems, 2000, vol. 114 pp. 477-484
[8] B. Schweizer and A. Sklar, Probabilistic Metric Space New York: Dover Publication, Inc., 2005 